\title{
Stumuli-Responsive Bio-Hybrid Nanogels: an Emerging Platform in Medicinal Arena
}

\author{
Prashant Sahu ${ }^{1}$, Sushil K Kashaw ${ }^{1,2 *}$, Samaresh Sau ${ }^{2}$ and Arun K Iyer ${ }^{2,3}$ \\ ${ }^{1}$ Department of Pharmaceutical Sciences, Dr. Harisingh Gour University (A Central University), India \\ ${ }^{2}$ Department of Pharmaceutical Sciences, Wayne State University, USA \\ ${ }^{3}$ Molecular Therapeutics Program, Wayne State University School of Medicine, USA
}

Submission: February 18, 2017; Published: March 14, 2017

*Corresponding author: Sushil K Kashaw, Department of Pharmaceutical Sciences, Wayne State University, USA; Email: sushilkashaw@gmail.com,

Keywords: Bio-hybrid; Cross-linked polymer; Biodegradability; Cancer; Nanogel; Nanotechnology

\section{Introduction}

Bio-hybrid nanogels are nano-sized hydrogels fabricated by physical or chemical cross linking under controlled conditions. The biodegradable nanogels functions as an eco-friendly carrier of drugs and bio actives for application in disease diagnosing, sensing and targeted therapy, with the added benefit of high patient compliance and safety.

The role of nanotechnology has become imminent in combating various lethal and local diseases along with highly desirable effect on disease diagnosis, imaging and treatment. More importantly, the impact of nano-medical technologies has been profound in the management of life threatening diseases such as cancer [1]. In this regard, several nano-based materials such as nano particles, dendrimers, liposomes and polymeric micelles have been widely researched and used for drug delivery. Along these lines, nanogels are gaining widespread attention and are been extensively characterized and evaluated for the management of several diseases in last few years.

The dose limiting toxicity and target tissue selectivity has been problematic in conventional chemotherapy and nanogels have great advantage in addressing these challenges [2]. In this regard, the utility of biodegradable materials such as natural and water soluble polymers can be very useful in mitigating the carrier related toxicity of the nano constructs. Bio-hybrid nanogels loaded with bioactives using such biodegradable materials when delivered topically, orally, parenterally or by intranasal routes transports the bio actives to the desired site and eradicates some of the delivery challenges associated with the free drug or stability of bioactive molecules such as peptides and genes [3].

The use of nanogels also guarantees transport of anticipated dose ofbio actives to the targeted site successfully thus eradicating the side effects and adverse complications of cytotoxic free drug administration. There cent developments and research on the compositions of nano carriers such as nano particles, niosomes, dendrimers and liposomes utilizing biodegradable functional groups, linkers and natural polymers can also facilitate the stimuli responsive triggered release of the bioactive at the site of action (such as tumors) thus enhancing the efficacy of treatment [4]. Thus, the current better understanding of the biology of the disease as well as the thorough knowledge on the construction and manipulation of bio-hybrid nanogels appear to have a high impact on improving the diagnostic and therapeutic efficiency of delivering such agents to diseases such as cancer [5].

\section{Biohybrid Nanogel}

Biohybrid water based nanogels systems are physically or chemically cross-linked colloids or micelles system encompassing hydrophilic polymer backbone. Biohybrid nanogel exhibits the features of bulk hydrogels system along with high stability of colloidal particles in aqueous dispersions [6]. Bio-hybrid nanogels can be easily dispersed in the organic or inorganic media, however, these nanogels are very delicate due to their organizational establishment. The gels can swell in aqueous medium and offer enhances level of drug loading. In addition, researchers have assessed that the hybrid nanogel prepared in an aqueous media by self-assembly of polymers 
are very efficient in rapid drug release to the targeted site [7]. High water content makes the nanogel system more amiable to transport in a fluid way which exhibits the molecule size less than a conventional gel system. Another versatile property of nanogel system is its stimuli responsive behavior like responsiveness to $\mathrm{pH}$, temperature, charge, enzyme activity or electrical/ electromagnetic pulse.

Nano size of gel system enhances the surface area which has significant benefit as a potential carrier for varieties of drugs, bio actives and chemotherapeutic agents [8]. The chemical constitution of nanogels are subject of great interest as the nature of the polymer or cross linking agents can play a vital role in their utility as a nano carrier for drug delivery. Biohybrid nanogels offer various characteristics and applications like biocompatibility, negligible tissue toxicity, benign towards biological pathways, high degree of solubility and site specific drug delivery. Bio hybrid nanogel system possess high surface to volume ratio of nanogel with fused bio molecules and chemical particles such as peptides, proteins, RNA, DNA that makes these "bio-like" system more receptive to disease diagnosing and treatment [9]. Bio-hybrid nano gel can be fabricated by various methods including chemical cross linking, W/O or 0/W modified emulsion method, photolithographic method and so forth, which shows high drug entrapment and encapsulation in aqueous media [10]. All these methods make use of monomers, cross linkers, reactive polymers unit and bio molecules filaments to initiate polymerization, complexation, and emulsification for the efficient synthesis of nanogel system [11].

The key properties of bio-hybrid nano gels system include their ability to combine bio molecules and chemical macromolecules complexed with physical or covalent bonds into 3D colloidal nano gel networks [12]. The vital features like meager tissue toxicity, degradability, stimuli responsive behavior and high solubility makes nano gels a powerful arsenal to combat virulent diseases such as cancers more effectively. The versatility of bio-hybrid nano gels in loading plethora of drugs molecules and bio-actives, their chemical and biological stability and sustained release characteristics are some exceptional features that makes them an ideal candidate for targeted drug delivery.

However, the complexity of the disease condition as well as the design criteria for engineering nano gels with desired properties remains a challenging task. Although so, with the advent of new initiatives and better understanding of the biology of the diseased state and the new knowledge on the utility of nano medical technologies portent that bio-hybrid nanogels are the emerging as potent drug carrier system in diagnosing, sensing, treatment and [13] management of several diseases and disorders in the medicinal arena [14].

\section{Outlook}

In the near future, bio-hybrid nano gels port ends to have great potentials and prospects for not only treating various diseased conditions but also for fulfilling other subset of goals. For instance, along with the drug delivery attributes of the existing bio-hybrid nano gels, we can re-be engineering the configuration of bio-hybrid nano gels, to accomplish co-delivery of a cocktail of drugs and bioactive agents. In addition, biohybrid-nano gels can be decorated with targeting peptides, ligands as well as imaging agents that can perform simultaneous disease diagnosis, imaging and therapy of diseases such as cancers. In addition, the nano gels can be fine-tuned to precisely deliver the drug in the targeted sites in a special and temporal fashion, by utilizing $\mathrm{pH}$ responsive, thermo sensitive, enzymatic or other stimuli response that in principle can revolutionize the field of personalized cancer therapy.

\section{References}

1. Yanasarn N, Sloat BR, Cui Z (2009) Nanoparticles engineered from lecithin-in-water emulsions as a potential delivery system for docetaxel. Int J Pharm 379(1): 174-180.

2. Vinogradov SV, Zeman AD, Batrakova EV, Kabanov AV (2005) Polyplex nanogel formulation for drug delivery of cytotoxic nucleoside analogs. J Controlled Release 107(1): 143-157.

3. Teskac K, Kristi J (2010) The evidence for solid lipid nanoparticles mediated cell uptake of Resveratrol. Int J Pharm 390(1): 61-69.

4. Sun B, Ranganathan B, Feng SS (2008) Multifunctional poly (D,Llactide-co-glycolide)/ montmorillonite PLGA/MMT nanoparticles decorated by Trastuzumab for targeted chemotherapy of breast cancer Biomaterials 29(4): 475-486.

5. Uhrich KE, Cannizzaro SM, Langer RS, Shakesheff KM (1999) Polymeric systems for controlled drug release. Chem. Rev 99(11): 3181-3198.

6. Singka GSL, Samah NA, Zulfakar MH, Yurdasipe A, Heard CM (2010) Enhanced topical delivery and anti-inflammatory activity of methotrexate from an activated nanogel. Eur J Pharm Biopharm 76(2): 275-281.

7. Sheihet L, Garbuzenko OB, Bushman J, Gounder MK, Minko T, et al. (2012) Paclitaxel in tyrosine-derived nanospheres as a potential anticancer agent, In vivo evaluation of toxicity and efficacy in comparison with paclitaxel in Cremophor. Eur J Pharm Sci 45(3): 320-329.

8. Shigemasa Y, Saito K, Sashiwa H, Saimoto H (1994). Enzymatic degradation of chitins and partially deacetylated chitins. Int J Biol Macromol 16(1): 43-49.

9. Sahu MK, Ahmad D (2010) Development and optimization of fixed dose antihypertensive combination drugs using double layer sustained release microsphere technology. Int Jour Pharm \& Biomed Res 1(5): 114-123.

10. Fabien Salaün, Isabelle Vroman (2009). Curcumin loaded nanocapsules, formulation and influence of the nanoencapsulation processes variables on the physico-chemical characteristics of the particles. Int J Chem React Eng 7(1): 1542-6580.

11. Passerini N, Craig DQM (2001) An investigation into the effects of residual water on the glass transition temperature of polylactide microspheres using modulated temperature DSC. J Control Release 73(1): 111-115.

12. Patnaik S, Sharma AK, Garg BS, Gandhi RP, Gupta KC (2007) Photoregulation of drug release in azo-dextran nanogels. Int J pharm 342(1-2): 184-193.

13. Li Y, Pei Y, Zhang X, Gu Z, Zhou Z, et al, (2001) PEGylated PLGA nanoparticles as protein carriers: Synthesis, preparation and biodistribution in rats. J Control Release 2001 71(2): 203-211. 
14. Wang Q Xu H, Yang X, Yang Y (2008) Drug release behavior from in situ gelatinized thermosensitive nanogel aqueous dispersions. Int J Pharm 361(1-2): 189-193.

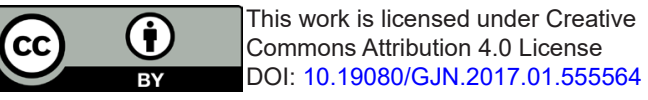

Your next submission with JuniperPublishers will reach you the below assets

- Quality Editorial service

- Swift Peer Review

- Reprints availability

- E-prints Service

- Manuscript Podcast for convenient understanding

- Global attainment for your research

- Manuscript accessibility in different formats ( Pdf, E-pub, Full Text, Audio)

- Unceasing customer service

Track the below URL for one-step submission https://juniperpublishers.com/submit-manuscript.php 